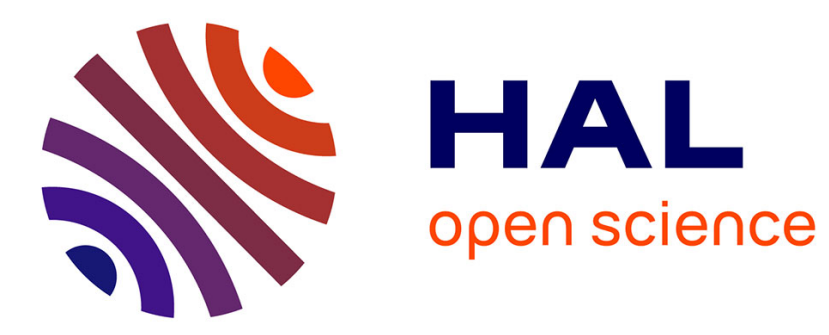

\title{
Direction of arrival estimation by a modified orthogonal propagator method with spline interpolation
}

\author{
Meng Sun, Yide Wang, Jingjing Pan
}

\section{To cite this version:}

Meng Sun, Yide Wang, Jingjing Pan. Direction of arrival estimation by a modified orthogonal propagator method with spline interpolation. IEEE Transactions on Vehicular Technology, 2019, 68 (11), pp.11389 - 11393. 10.1109/TVT.2019.2944516 . hal-02308199

\section{HAL Id: hal-02308199 \\ https://hal.science/hal-02308199}

Submitted on 9 Dec 2019

HAL is a multi-disciplinary open access archive for the deposit and dissemination of scientific research documents, whether they are published or not. The documents may come from teaching and research institutions in France or abroad, or from public or private research centers.
L'archive ouverte pluridisciplinaire HAL, est destinée au dépôt et à la diffusion de documents scientifiques de niveau recherche, publiés ou non, émanant des établissements d'enseignement et de recherche français ou étrangers, des laboratoires publics ou privés. 


\title{
Direction of arrival estimation by a modified orthogonal propagator method with spline interpolation
}

\author{
Meng Sun, Yide Wang, Jingjing Pan
}

\begin{abstract}
This paper presents a modified orthogonal propagator method (OPM) with spline interpolation for direction of arrival (DoA) estimation. The conventional OPM is based on the noise-free signal model. When noise exists, the main contribution of noise (additive temporally and spatially white Gaussian noise) is in the diagonal elements of the data covariance matrix. Therefore, the influence of noise can be minimized by restoring the noise free diagonal elements through an interpolation procedure and the propagator can then be directly extracted from the denoised data covariance matrix, which is the main idea of the proposed method in this paper. The performance of the proposed modified OPM is tested through numerical simulations. Simulation results show that the proposed modified OPM achieves low root-mean square errors (RMSEs) with small computational load in DoA estimation.
\end{abstract}

Index Terms-Direction of arrival (DoA), orthogonal propagator method (OPM), propagator method (PM), spline interpolation, denoising.

\section{INTRODUCTION}

Direction of arrival (DoA) estimation is an important issue in the field of array signal processing and has been intensively studied for decades [1], [2]. It is important in many practical applications such as sonar, radar, source localization, and wireless communication [1], [2]. A lot of methods have been developed for DoA estimation in the literature. Among these methods, subspace-based algorithms or high-resolution methods such as MUSIC [3] and ESPRIT [4] have attracted much attention due to their asymptotic infinite resolution and unbiased estimation performance. These methods are based on the assumption of the orthogonality between the signal subspace and noise subspace of the received signals. However, the above techniques require either the eigenvalue decomposition (EVD) or singular value decomposition (SVD) of the data covariance matrix. It may be too expensive and time-consuming for real time applications with a large amount of data, such as a massive Multiple-Input Multiple-Output system with a very large number of the antennas (hundreds or thousands). Besides, this kind of methods may have some limitation due to the high computational complexity, in some practical scenarios, for example, long time field work without guaranteed electronic energy.

To solve this problem, a concept named 'propagator' was introduced in array signal processing. Many methods have been proposed based on this concept, such as propagator method (PM) [5], [6] and orthogonal propagator method (OPM) [7]. These methods only use linear operations to find the propagator (which can be directly extracted through the data covariance matrix), without any EVD or SVD operation. The propagator has been proven effective in DoA estimation. Besides, the propagator-based methods are more suitable for dealing with large data, which can facilitate real time application. Nevertheless, the initial propagator-based algorithm does not take the background noise into consideration, as its basis relies on the noise free received signal model. Therefore, its performance suffers from degradation in low signal to noise ratio (SNR) scenarios. The least squares method is applicable in the estimation of the propagator, which reduces the impact of noise but does not eliminate it [5].

Researchers attempt to reduce the noise impact by estimating the power of the noise [8], [9], [10] or applying signal enhancement techniques [11], [12]. Stoica et al. propose an eigenvalue-based method [8] to estimate the noise power, which requires the EVD on the data covariance matrix. The noise power is estimated by calculating the average of the eigenvalues belonging to the noise subspace. The eigenvalue method is proven to be statistically efficient [8]. However, it is not the focus of this paper since the major merit of the propagator-based methods is to avoid any EVD or SVD operation. In addition, Stoica et al. [8], Marcos and SanchezAraujo [6] also propose two different linear methods. In their methods, the noise power can be extracted from the structure of the data covariance matrix without EVD or SVD operation. These two linear methods are more computationally efficient, yet less statistically efficient, compared with the eigenvalue based methods [8].

In this paper, a new way to reduce the influence of noise without estimating the noise power is proposed from another point of view. The noise is assumed as an additive temporally and spatially white Gaussian noise. The noise hence will only affect the main diagonal elements of the data covariance matrix. Therefore, we propose to restore the noise free diagonal elements of the data covariance matrix to reduce the influence of the noise. In a column (or line), the diagonal element can be estimated by the other elements of this column (or line), which could be done by an interpolation procedure. It is worth noting that interpolation techniques have been widely used in array signal processing. In [2], array interpolation is used to map the sample covariance matrix of a non-uniform linear array to that of a uniform linear array, so that root-MUSIC and ESPRIT can be directly applied. Instead, the interpolation procedure in this paper is used to rebuild the noise free diagonal elements of the data covariance matrix. Moreover, when the incoming signals are uncorrelated or independent, the data covariance matrix is a 
Toeplitz matrix, which means that the diagonal elements of this matrix are all the same [13]. Consequently, the interpolation needs to be performed only once to recover all the noise free diagonal elements of the matrix. In this paper, the cubic spline interpolation [14] is adopted. The performance of the proposed modified OPM algorithm is evaluated on numerical data.

The remaining of this paper is organised as follows. The narrow band received signal model in far-field is presented in Section 2. In Section 3, the proposed modified OPM is introduced. Section 4 gives the simulation results to show the performance of the proposed method compared with the conventional OPM, OPM with elimination of noise called MPEN (method of propagator with elimination of noise) [6], OPM with EVD [8] and MUSIC [3]. Section 5 draws the conclusion.

\section{SignAL MODEL}

Consider a uniform linear array (ULA) with $N=$ $2 M+1$ isotropic antennas impinged by $K$ far-field narrow band independent signals. The antennas are indexed by $-M, \ldots, 0, \ldots, M$. The received signals are corrupted by a temporally and spatially white Gaussian noise. In the following, the 0th element is taken as the reference point. Then, the complex envelope of the received signal at the $m$ th antenna can be expressed as

$$
r_{m}(t)=\sum_{k=1}^{K} s_{k}(t) \exp \left(-j 2 m \pi \frac{d}{\lambda} \sin \theta_{k}\right)+n_{m}(t)
$$

where $s_{k}(t)$ is the complex envelope of the $k$ th signal received at the reference point with corresponding DoA $\theta_{k}$ $\left(\theta_{k} \in[-\pi / 2, \pi / 2]\right) ; n_{m}(t)$ is the spatially and temporally additive white Gaussian noise at the $m$ th antenna with zero mean and variance $\sigma^{2} ; d$ denotes the distance between two adjacent antenna and $\lambda$ is the wavelength of the received signals. $d$ is equal to half of the wavelength of the incoming signals.

Equation (1) can be written in the following vector form:

$$
\mathbf{r}(t)=\mathbf{A s}(t)+\mathbf{n}(t)
$$

with the following notational definitions:

- $\mathbf{r}(t)=\left[r_{-M}(t) r_{-M+1}(t) \cdots r_{0}(t) \cdots r_{M}(t)\right]^{T}$ is the $(N \times 1)$ received signal vector or the observation vector; the superscript $T$ denotes the transpose operation;

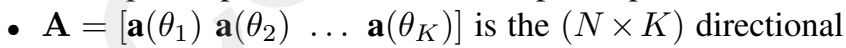
matrix;

- $\mathbf{a}\left(\theta_{k}\right)=\left[\exp \left(j M \pi \sin \theta_{k}\right) \ldots 1 \ldots \exp \left(-j M \pi \sin \theta_{k}\right)\right]^{T}$ is the $(N \times 1)$ directional vector;

- $\mathbf{s}(t)=\left[\begin{array}{llll}s_{1}(t) & s_{2}(t) & \cdots & s_{K}(t)\end{array}\right]^{T}$ is the $(K \times 1)$ source vector;

- $\mathbf{n}(t)=\left[n_{-M}(t) n_{-M+1}(t) \cdots n_{0}(t) \cdots n_{M}(t)\right]^{T}$ is the $(N \times 1)$ noise vector, with zero mean and covariance matrix $\sigma^{2} \mathbf{I}$, where $\mathbf{I}$ is the $(N \times N)$ identity matrix.

Assuming that the noise is independent of the source signals, the covariance matrix $\mathbf{R}$ can be written as:

$$
\mathbf{R}=E\left(\mathbf{r}(t) \mathbf{r}^{H}(t)\right)=\mathbf{A R}_{s} \mathbf{A}^{H}+\sigma^{2} \mathbf{I}
$$

where $E(:)$ denotes the ensemble average; $\mathbf{R}_{s}$ is the $(K \times K)$ dimensional covariance matrix of the source vector $\mathbf{s}(t)$ and the superscript $H$ denotes the complex conjugate transpose operation.

\section{MODIFIED OPM FOR DOA ESTIMATION}

In order to reduce the noise impact, OPM-MPEN is proposed [6]. However, this method can only localize a maximum of $N / 2-1$ sources. In this section, we will present a new modified OPM algorithm which avoids such limitation. The signals are supposed to be totally uncorrelated to each other and independent from the noise. The elements in $\mathbf{R}$ can then be expressed as follows:

$R(n-m)=\mathbf{R}(m, n)= \begin{cases}\sum_{k=1}^{K} P_{s_{k}}+\sigma^{2} & m=n \\ \sum_{k=1}^{K} P_{s_{k}} \exp \left(j \pi(n-m) \sin \theta_{k}\right) & m \neq n\end{cases}$

where $m, n \in[1, \ldots N], P_{s_{k}}$ is the power of the $k$ th signal. $R(n-m)$ is a function of $n-m$, which represents the expression of the element of the $m$ th row and $n$th column of matrix $\mathbf{R}$. From (4), the noise is presented only in the diagonal elements of the covariance matrix $\mathbf{R}$.

However, with finite snapshots, the non-diagonal elements contain residual from noise. In order to show the noise impact on both the diagonal and non-diagonal elements of the data covariance matrix with different snapshots, we define the noise uncertainty ratios (NURs) as the ratio between the elements $y_{m n}$ of the noise free data covariance matrix $\mathbf{A R}_{s} \mathbf{A}^{H}$ and the elements $z_{m n}$ of matrix $\hat{\mathbf{R}}-\mathbf{A} \mathbf{R}_{s} \mathbf{A}^{H}$ (for non-diagonal elements, NURs= $\left\{\sum_{m=1}^{N} \sum_{n=m+1}^{N}\left|y_{m n}\right|\right\} /\left\{\sum_{m=1}^{N} \sum_{n=m+1}^{N}\left|z_{m n}\right|\right\}$; for diagonal elements, NURs $\left.=\left\{\sum_{m=1}^{N}\left|y_{m m}\right|\right\} /\left\{\sum_{m=1}^{N}\left|z_{m m}\right|\right\}\right)$, where $\hat{\mathbf{R}}=\frac{1}{N_{t}} \sum_{i=1}^{N_{t}} \mathbf{r}\left(t_{i}\right) \mathbf{r}^{H}\left(t_{i}\right), N_{t}$ is the number of snapshots. NURs on both diagonal and non-diagonal elements of the data covariance matrix are calculated in terms of the number of snapshots with 1000 independent Monte-Carlo trials and $\mathrm{SNR}=0 \mathrm{~dB}$.

It can be seen from Fig. 1, for both the diagonal and nondiagonal elements, the influence of the noise decreases (NURs increases) when the number of snapshots increases. Moreover, the influence of the noise is greater (smaller NURs) to the diagonal elements than to the non-diagonal elements, especially when the number of snapshots is large. Therefore, we focus on the diagonal elements and the influence of the noise could be reduced by rebuilding the noise free diagonal elements from the non-diagonal elements of the data covariance matrix.

The noise free diagonal elements can be estimated by the other non-diagonal elements. Define $f(x)=R(x)=$ $\sum_{k=1}^{K} P_{s_{k}} \exp \left(j \pi x \sin \theta_{k}\right)$ with $x=n-m$, therefore $x \in$ $[-N+1, N-1]$. When $x=0, f(0)=\sum_{k=1}^{K} P_{s_{k}}$ corresponding to the value of the noise free diagonal element (unknown). $f(0)$ can be estimated from its adjacent elements of $f(x)$ (or off-diagonal elements $R(x)), x \in[-N+1,-1] \bigcup[1, N-1]$ by the classical spline interpolation procedure [14]. In addition, as $\mathbf{R}$ is a Toeplitz matrix, the diagonal elements of 


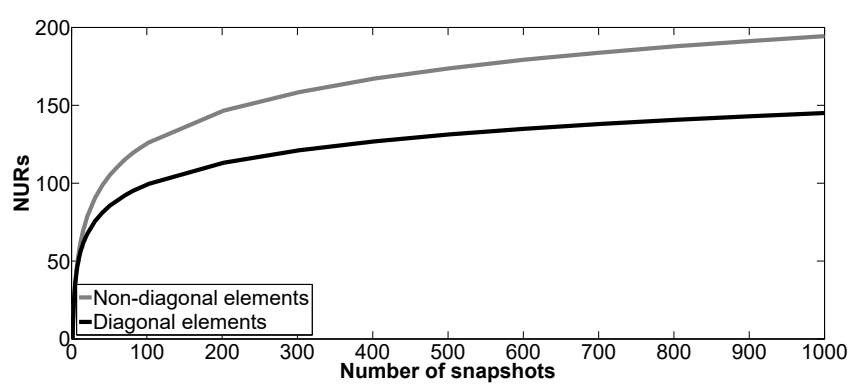

Fig. 1. NURs on both the diagonal and non-diagonal elements of the data covariance matrix versus the number of snapshots with 1000 independent Monte-Carlo trials, SNR of the received signal equals to $0 \mathrm{~dB}$.

this matrix are all equal. Therefore, it is enough to estimate only one noise free diagonal element. As the variation of the norm of non-diagonal elements is slower than that of the elements themselves, in this paper, the spline interpolation is applied on the norm of the non-diagonal elements in the first column and first line of the data covariance matrix for the calculation of the corresponding noise free diagonal element $f(0)$. It means by using $|f(-N+1)|, \mid f(-N+$ $2)|, \ldots| f,(-1)|| f,(1)|\ldots| f,(N-1) \mid$, we can estimate the noise free diagonal element $f(0)$ through spline interpolation. The detail is given as follows: to estimate the expression of $|f(x)|$, the spline function $S(x)$ is built with $2 N-2$ sample points $(-N+1, \ldots,-1,1, \ldots, N-1)$ and $2 N-3$ intervals. $S_{i}(x)$ is the spline function inside the $i$ th interval. $S(x)$ should obey the following constrains:

- In each interval, $S(x)=S_{i}(x)$ is a three order polynomial.

- $S(x)$ and its derivatives $S^{\prime}(x), S^{\prime \prime}(x)$ should be continuous for $x \in(-N+1, N-1)$.

- $S\left(x_{i}\right)=\left|f\left(x_{i}\right)\right|, x_{i}=-N+1, \ldots,-1,1, \ldots, N-1$.

- $S^{\prime \prime \prime}(-N+1)=S^{\prime \prime \prime}(-N+2)$ and $S^{\prime \prime \prime}(N-1)=S^{\prime \prime \prime}(N-$ 2).

The principle of spline interpolation is to take a shape that minimizes the bending (under the constraint of passing through all sample points). Both $S^{\prime}(x)$ and $S^{\prime \prime}(x)$ should be continuous for $x \in(-N+1, N-1)$. The above can be achieved with polynomials of degree 3 or higher. The classical spline interpolation is to use polynomials of degree 3 [14]. Therefore, $S_{i}(x)$ can be expressed as $S_{i}(x)=a_{i} x^{3}+b_{i} x^{2}+c_{i} x+g_{i}$, with $a_{i}, b_{i}, c_{i}, g_{i}$ unknown coefficients. By using the principle of the spline interpolation, the $8 N-12$ unknown coefficients inside $S(x)$ can be calculated. Then, the spline function $S(x)$ can be rebuilt. Consequently, the noise free diagonal elements can be estimated by $S(0)$, which is used to restore all the diagonal elements of $\mathbf{R}$. The elements of the denoised data covariance matrix $\mathbf{R}_{n f}$ can then be written as

$$
\mathbf{R}_{n f}(m, n)=\sum_{k=1}^{K} P_{s_{k}} \exp \left(j \pi(n-m) \sin \theta_{k}\right) .
$$

Since the noise is removed, OPM can be directly applied on the denoised data covariance matrix for DoA estimation. OPM
[7] partitions matrix $\mathbf{A}$ into two sub-matrices as follows:

$$
\mathbf{A}=\left(\begin{array}{c}
\mathbf{A}_{1} \\
\mathbf{A}_{2}
\end{array}\right)
$$

where $\mathbf{A}_{1}$ and $\mathbf{A}_{2}$ are $(K \times K)$ and $((N-K) \times K)$ dimensional matrices $(N>K)$, respectively. Since $\theta_{k} \in[-\pi / 2, \pi / 2]$ (for any $\theta_{i}, \theta_{j} \in[-\pi / 2, \pi / 2]$, if $\left.\theta_{i} \neq \theta_{j}, \sin \theta_{i} \neq \sin \theta_{j}\right), \mathbf{A}$ is of full rank and $\mathbf{A}_{1}$ is non-singular. Under the above condition, the following $(K \times(N-K))$ dimensional propagator $\mathbf{P}$ can be defined

$$
\mathbf{P}^{H} \mathbf{A}_{1}=\mathbf{A}_{2}
$$

or

$$
\left[\mathbf{P}^{H},-\mathbf{I}_{N-K}\right] \mathbf{A}=\mathbf{Q}^{H} \mathbf{A}=\mathbf{0}
$$

where $\mathbf{I}_{N-K}$ is a $((N-K) \times(N-K))$ dimensional identity matrix. Equation (8) shows that the directional vectors of sources are orthogonal to the columns of $\mathbf{Q}$. Therefore, the DoA of each source can be estimated by searching the peak positions of the following OPM pseudo-spectrum:

$$
P_{\mathrm{OPM}}(\theta)=\frac{1}{\mathbf{a}^{H}(\theta) \mathbf{Q}\left(\mathbf{Q}^{H} \mathbf{Q}\right)^{-1} \mathbf{Q}^{H} \mathbf{a}(\theta)} .
$$

However, propagator $\mathbf{P}$ is unknown, which should be retrieved from data.

Firstly, the data covariance matrix is partitioned into the following expression:

$$
\mathbf{R}_{n f}=[\mathbf{G}, \mathbf{H}]
$$

where $\mathbf{G}$ and $\mathbf{H}$ are the $(N \times K)$ and $(N \times(N-K))$ dimensional matrices, respectively. The relationship between propagator $\mathbf{P}$ and these two matrices $(\mathbf{G}$ and $\mathbf{H}$ ) is given by

$$
\mathbf{H}=\mathbf{G P} \text {. }
$$

Therefore, propagator $\mathbf{P}$ can be directly extracted through (11) by the least squares solution:

$$
\mathbf{P}=\mathbf{G}^{+} \mathbf{H}
$$

where the superscript + denotes the Moore-Penrose inverse operator.

Finally, the general steps of the proposed modified OPM are summarized as follows:

1) Estimate the covariance matrix $\hat{\mathbf{R}}(3)$.

2) Make the estimated covariance matrix $\hat{\mathbf{R}}$ Toeplitz, denoted by $\mathbf{R}_{T}$. The Hermitian Toeplitz matrix $\mathbf{R}_{T}$ can be built by calculating the first line of this matrix, $r_{t}(m)=$ $\frac{1}{N-m} \sum_{n=1}^{N-m} \hat{\mathbf{R}}(n, m+n)$, with $m=1, \ldots, N-1$.

3) Apply the spline interpolation on the norm of the $4 M$ non-diagonal elements (the norm of the elements) of the first column and the first line of $\mathbf{R}_{T}$ to estimate its diagonal element.

4) Construct the new data covariance matrix $\mathbf{R}_{n f}$ by replacing the diagonal elements of $\mathbf{R}_{T}$ with the above estimated diagonal element.

5) Apply the OPM on the new data covariance matrix $\mathbf{R}_{n f}$ to estimate the DoA. 


\section{Simulations AND Discussion}

The performance of the proposed modified OPM is evaluated on numerical data with 4 simulations. Considering a ULA with 11 isotropic elements and 2 far-field narrow band uncorrelated signals with equal power, that is, $N=11, M=5$ and $K=2$.

In the first simulation, the pseudo-spectrums of the conventional OPM, OPM-MPEN and proposed method are compared. The following two cases are considered:

- Case 1. $\theta_{1}=-6^{\circ}$ and $\theta_{2}=7^{\circ}$.

- Case 2. $\theta_{1}=-1^{\circ}$ and $\theta_{2}=3^{\circ}$.

The data covariance matrix is estimated with 400 independent snapshots and $\mathrm{SNR}=0 \mathrm{~dB}$. The pseudo-spectrum search is performed over $\left[-15^{\circ}, 15^{\circ}\right]$ with step size $0.001^{\circ}$. The angles corresponding to the two highest peak positions in the pseudospectrum allow estimating the DoAs of signals.

Figs. 2-3 show the pseudo-spectrums of the conventional OPM, OPM-MPEN and proposed method for the two different cases. For both cases, the proposed method is able to detect the true DoAs of signals. As shown in Figs. 2-3, two peaks corresponding to the DoAs of signals are clearly detected by the proposed method. For the conventional OPM and OPM-MPEN, they can detect the true DoAs of signals in large angle separation situation (in Case 1) and their pseudospectrums are similar to that of the proposed method. However, when the angle separation becomes small, like in Case 2, the conventional OPM fails in DoA estimation and the OPMMPEN estimates the DoA with bias.

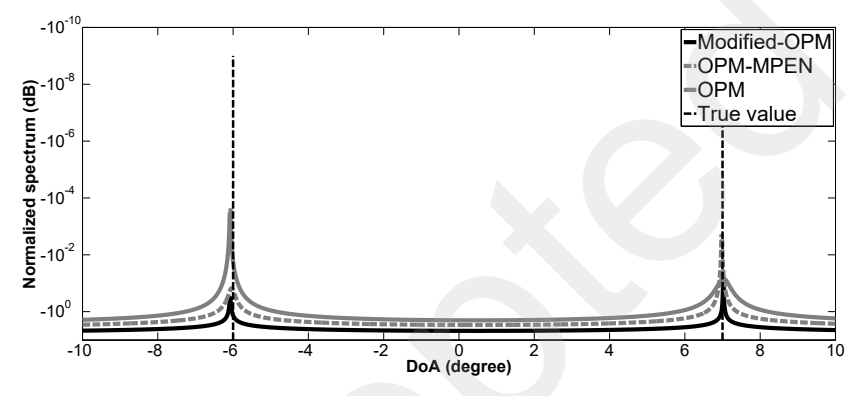

Fig. 2. Case 1, pseudo-spectrums of the conventional OPM, OPM-MPEN and proposed modified OPM for DoA estimation. The two DoAs are $\theta_{1}=-6^{\circ}$ and $\theta_{2}=7^{\circ}$.

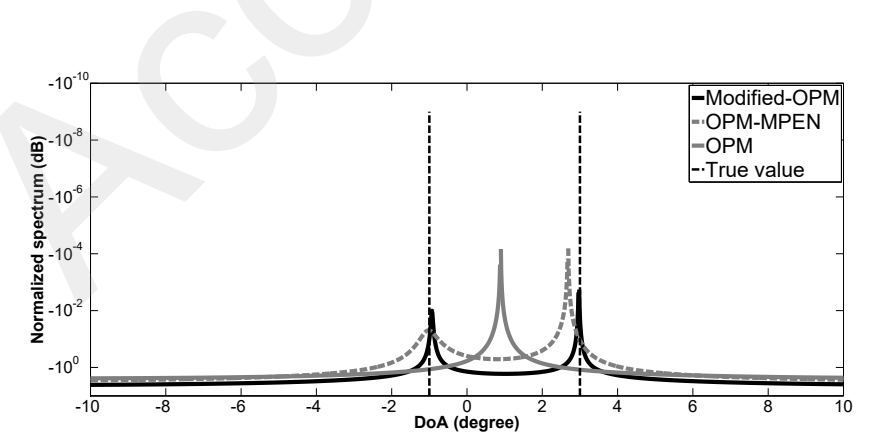

Fig. 3. Case 2, pseudo-spectrums of the conventional OPM, OPM-MPEN and proposed modified OPM for DoA estimation. The two DoAs are $\theta_{1}=-1^{\circ}$ and $\theta_{2}=3^{\circ}$.
In the second simulation, the performance of the proposed method is tested with respect to SNR. A Monte-Carlo process is constructed with 1000 independent runs of the method. The performance is assessed in terms of the RMSE of the estimated DoA defined as

$$
\mathrm{RMSE}=\sqrt{\frac{1}{K J} \sum_{k=1}^{K} \sum_{j=1}^{J}\left(\hat{\theta}_{k j}-\theta_{k}\right)^{2}}
$$

where $\hat{\theta}_{k j}$ denotes the estimated DoA of the $k$ th incoming signal for the $j$ th run; $\theta_{k}$ denotes the true DoA of the $k$ th incoming signal. The angles in Case 2 are used here. SNR $\in[-6,10] \mathrm{dB}$. The proposed method is compared with OPMMPEN, OPM-EVD and MUSIC; moreover, the Cramér-Rao bound (CRB) results are also provided [15], [16].

Fig. 4 plots the RMSE of DoA estimation against SNR with the number of snapshots equals to 400 . As expected, the RMSEs of the above mentioned methods continuously decrease when SNR increases. According to the principle of OPM [7], the propagator based methods are efficient in terms of computational burdens, but their statistical performance is worse than that of the subspace based methods. Therefore, the gap (estimation performance) between the CRB and propagator based methods can be large. Same results can be found in Fig. 4 that the RMSE of MUSIC versus SNR is smaller than that of the propagator based methods at the beginning of SNR. However, with higher SNR $(-2 \mathrm{~dB})$, these methods tend to work similarly. In addition, MUSIC applies EVD operation with computational load $O\left(N^{3}\right)$, which is greater than that of OPM $\left(O\left(N^{2} K+(N-K)^{2} K\right)\right.$, DoA spectrum search is not taken into account in computational complexity).

Compared with the other denoising procedures, the proposed method has a more significant decrease of RMSE than that of the OPM-MPEN; its performance is similar to that of OPM-EVD. The computational loads of the denosing procedures of OPM-MPEN, OPM-EVD, and the proposed method are shown in Table I. From Table I, the proposed method has the smallest computational complexity among the 3 compared methods.

\begin{tabular}{|c|c|}
\hline Method & Computational loads \\
\hline OPM-MPEN & $O\left((N-K) K^{2}+K^{3} / 3\right)$ \\
\hline OPM-EVD & $O\left(N^{3}\right)$ \\
\hline Modified OPM & $O(N+\log N)$ \\
\hline
\end{tabular}

THE COMPUTATIONAL LOADS OF THE DENOSING PROCEDURES OF OPM-MPEN, OPM-EVD AND MODIFIED OPM.

In the third simulation, the statistical performance of the modified OPM versus the angle separation between two incoming signals is assessed with a Monte-Carlo process of 1000 independent runs of the method. One of the incoming signals is fixed at $\theta_{1}=0^{\circ}$ while the other comes from $\theta_{2}=\theta_{1}+\Delta \theta$ with same power. SNR $=0 \mathrm{~dB}, \Delta \theta$ varies from $0.5^{\circ}$ to $8^{\circ}$. The number of snapshots is 400 . From Fig. 5, it is clear that the mentioned methods fail to detect the DoAs when the angle separation is small $\left(0.5^{\circ}\right.$ to $\left.2^{\circ}\right)$. The performance of the proposed methods is similar to that of the second simulation. 


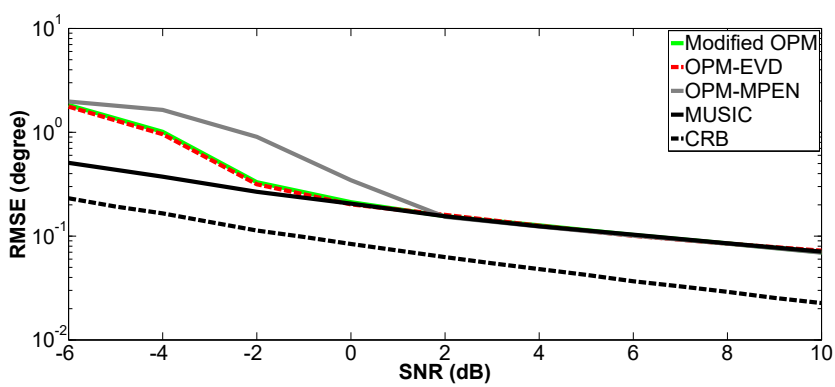

Fig. 4. Case 2, RMSE of DoA estimation versus SNR with 400 snapshots.

Moreover, the RMSEs of the mentioned methods continuously decrease as the angle separation increases. When the angle separation becomes greater, these methods tend to have similar performance.

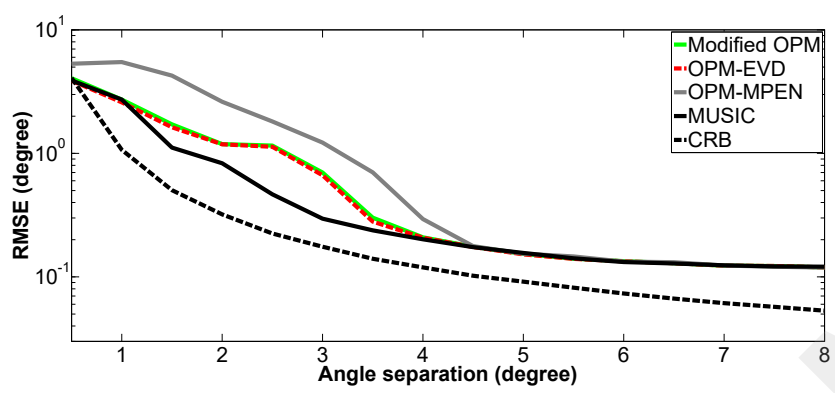

Fig. 5. RMSE of DoA estimation as function of angle separation.

Moreover, the performance of the proposed modified OPM versus the number of snapshots is evaluated with a numerical example of 1000 independent Monte-Carlo trials. Case 2 is studied with $\mathrm{SNR}=0 \mathrm{~dB}$. Fig. 6 plots the RMSEs of the modified OPM, OPM-MPEN, OPM-EVD and MUSIC as function of the number of snapshots. In Fig. 6, the above mentioned methods fail when the number of snapshots is small (less than 10). With the increasing number of snapshots, the RMSEs decrease. Different from MUSIC, we can observe that the propagator based methods require good estimation of the data covariance matrix with a sufficient number of snapshots $(>50)$.

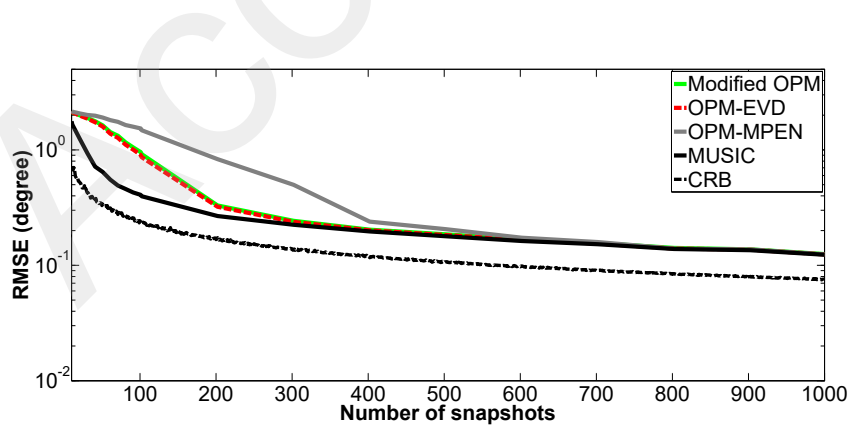

Fig. 6. RMSE of DoA estimation as function of the number of snapshots, $\mathrm{SNR}=0 \mathrm{~dB}$.

\section{CONCLUSION}

In this paper, we propose a modified OPM method for DoA estimation. By using the spline interpolation to rebuild the noise free diagonal elements of the data covariance matrix, the proposed method offers a new way to reduce the noise impact. The performance of the proposed method is tested on numerical data. The proposed modified OPM shows good performance in DoA estimation.

\section{ACKNOWLEDGEMENT}

The authors would like to thank the Shanghai Sailing Program (No.19YF1419100) and the National Natural Science Foundation of China (No.61701297) for funding part of this work.

\section{REFERENCES}

[1] H. Krim and M. Viberg, "Two decades of array signal processing research: the parametric approach," IEEE Signal Processing Magazine, vol. 13, no. 4, pp. 67-94, 1996.

[2] T. E. Tuncer and B. Friedlander, "Narrowband and wideband DOA estimation for uniform and non-uniform linear arrays," Classical and Modern Direction-of-Arrival Estimation, pp. 138-173, 2009.

[3] R. O. Schmidt, "Multiple emitter location and signal parameter estimation," International Journal of Engineering Research, vol. 34, no. 3 , pp. 276-280, 1986.

[4] R. Roy and T. Kailath, "ESPRIT-estimation of signal parameters via rotational invariance techniques," IEEE Transactions on Acoustics, Speech, and Signal Processing, vol. 37, no. 7, pp. 984-995, 1989.

[5] J. Munier and G. Y. Delisle, "Spatial analysis using new properties of the cross-spectral matrix," IEEE Transactions on Signal Processing, vol. 39, no. 3, pp. 746-749, 1991.

[6] S. Marcos and J. Sanchez-Araujo, "Méthodes linéaires haute résolution pour l'estimation de directions d'arrivée de sources. performances asymptotiques et complexité," Traitement du signal, vol. 14, no. 2, pp. 99-116, 1997.

[7] S. Marcos, A. Marsal, and M. Benidir, "The propagator method for source bearing estimation," Signal Processing, vol. 42, no. 2, pp. 121138,1995

[8] P. Stoica, T. Sderstrm, and V. ك̆ imonyte, "On estimating the noise power in array processing," Signal Processing, vol. 26, no. 2, pp. 205-220, 1992.

[9] M. Morelli and M. Moretti, "Joint maximum likelihood estimation of CFO, noise power, and SNR in OFDM systems," IEEE Wireless Communications Letters, vol. 2, no. 1, pp. 42-45, 2013.

[10] Y. Liu, G. Y. Li, Z. Tan, and H. Hu, "Noise power estimation in SCFDMA systems," IEEE Wireless Communications Letters, vol. 4, no. 2, pp. 217-220, 2017.

[11] J. Li, C. L. Bastard, Y. Wang, G. Wei, B. Ma, and M. Sun, "Enhanced GPR signal for layered media time-delay estimation in low-SNR scenario," IEEE Geoscience and Remote Sensing Letters, vol. 13, no. 3, pp. 299-303, 2016.

[12] I. Volaric, V. Sucic, and S. Stankovic, "A data driven compressive sensing approach for time-frequency signal enhancement," Signal Processing, vol. 141, pp. 229-239, 2017.

[13] R. A. Horn, Topics in matrix analysis. Cambridge University Press, 1994.

[14] L. L. Schumaker, Spline functions:basic theory. Cambridge University Press, 1981.

[15] P. Stoica and N. Arye, "MUSIC, maximum likelihood, and cramér-rao bound," IEEE Transactions on Acoustics, Speech, and Signal Processing, vol. 38, no. 12, pp. 2140-2150, 1990.

[16] P. Stoica, E. G. Larsson, and A. B. Gershman, "The stochastic crb for array processing: A textbook derivation," IEEE Signal Processing Letters, vol. 8, no. 5, pp. 148-150, 2001. 